\title{
Modelo matricial de Investigaciones macroeconómicas simultáneas, orientadas al diseño de políticas públicas
}

\author{
Matrix model of simultaneous macroeconomic research for design \\ of public policies
}

Artículo recibido en septiembre 2020

Arbitrado en octubre 2020

Aceptado en octubre 2020

Publicado en enero 2021

\section{Luis Arturo Franco Soliz \\ luarfrancos@gmail.com \\ ORCID: 0000-0002-6792-5679}

Instituto de Planificación y Prospección Estratégica

La Paz - Bolivia
Palabras clave

ABSTRACT

Keywords
El objetivo de este artículo científico fue proponer un modelo que permita elevar la cantidad, pertinencia y calidad de investigaciones científicas que se desarrollan en las universidades, orientadas a la generación de Políticas Públicas en Bolivia. El estudio se focalizó en la Unidad de Postgrado de la Facultad de Economía de la Universidad Mayor de San Andrés. Se trata de un estudio cualitativo de corte transversal, de alcance exploratorio y descriptivo que involucró la aplicación de encuestas a estudiantes, entrevistas a docentes eméritos y consulta a líderes empresariales bolivianos. Como resultado se presentó un modelo matricial de investigaciones, que estableció su factibilidad, en dirección a que las investigaciones académicas macroeconómicas sean un aporte al diseño de Políticas Públicas, impacten en el incremento de éstas y encuentren una mejor estructura para su financiamiento.

Modelo matricial; investigación multidisciplinar; enfoque interdisciplinar; vinculación académica; políticas públicas; macroeconomía 


\section{INTRODUCCIÓN}

La historia de la humanidad constata que el hombre busca permanentemente la justificación de la ocurrencia de fenómenos en su entorno, y ha alcanzado hallazgos en su afán e interés de responder a problemas trascendentales (Bosque-Rodríguez 1998). La incertidumbre entonces no es una debilidad, por el contrario, es una fortaleza del razonamiento científico que motiva a la investigación (Anguera, 1996). Se entiende que la investigación científica debería ser un estudio sistemático, controlado, reflexivo y crítico de supuestas relaciones que existen entre fenómenos naturales, para adquirir conocimientos y resolver problemas (Lases \& Robles, 2008). En este sentido, Vargas (2009) enfatiza en que la investigación retroalimenta el ejercicio de la profesión $y$, por tanto, considera que es imprescindible posibilitar espacios de generación de conocimiento en la formación académica. Gómez (2011) aporta en el análisis y postula que la investigación, la docencia y la extensión no pueden estar separadas, sino más bien deben articularse como un todo. En este sentido, el aprendizaje deberá articular la metodología, la epistemología, la filosofía organizacional y el enfoque sociopolítico.

En la actualidad, en los países desarrollados existe una notable relación entre inversión en investigación científica y crecimiento económico, traducida en presupuestos consistentes destinados a la creación de institutos de investigación que patentizan la calidad y puesta en marcha de sus trabajos, a través de su influencia notoria y creciente en la definición de Políticas Públicas y su aplicación en planes estratégicos de desarrollo. La participación de las universidades para la creación de conocimiento es activa y estratégica.

Esta realidad no es la misma en todos los países del mundo. En Bolivia, país de economía emergente, la asignación de presupuestos para la investigación científica es baja. En las casas superiores de estudio, tanto públicas como privadas, los esfuerzos son tan escasos que se ha confundido el concepto de construcción de la ciencia y la identificación de los problemas de la sociedad, así como sus alternativas de solución, en simples recopilaciones que en la mayoría de los casos matizan soluciones ya conocidas que han fracasado en su ejecución, desnudando falta de impacto social y evidenciando errores notables que son producto de su inconsistencia científica.

En consecuencia, si los recursos asignados a la investigación científica dentro de las universidades no son suficientes, y los pocos disponibles son aplicados en trabajos que se realizan erróneamente al cobijo del concepto y significado de ciencia; sin llegar a constituir auténticos trabajos de investigación científica, se detecta un profundo y grave problema para la sociedad; por lo tanto, es importante generar a través del pensamiento crítico, una nueva forma de ejecutar proyectos de investigación científica, buscando su impacto en la calidad de vida con la orientación de encontrar alternativas y soluciones posibles a los problemas y necesidades de la sociedad, en relación a los métodos científicos.

El origen y la motivación de este artículo científico se focalizó en la Unidad de Postgrado de la Facultad de Economía de la Universidad 
Mayor de San Andrés (UMSA), en este sentido el objetivo planteado fue proponer un modelo que permita incrementar la cantidad, pertinencia y calidad de investigaciones científicas, necesarias para orientar las Políticas Públicas del sector de la economía nacional, maximizando el beneficio y la gestión de los recursos actuales y potenciales, destinados a la investigación científica académica.

Dada la escasa producción científica vinculada al crecimiento económico en Bolivia, la pertinencia de este estudio recae en buscar la factibilidad de una propuesta de ejecución de investigaciones científicas académicas, relacionadas en torno a una problemática macroeconómica y su realización simultánea dentro de un modelo matricial, que permite racionalizar la utilización eficiente de recursos humanos calificados con experticia. En este sentido, se planteó consultar y estudiar a la población de investigadores potenciales egresados de los distintos programas de postgrado que tienen pendiente cumplir con la fase de investigación para titularse, para determinar la factibilidad de contar con su participación bajo los enfoques multidisciplinar e interdisciplinar, con orientación de complementariedad. Asimismo, se estudió los recursos tecnológicos, financieros, logísticos y otros necesarios para el desarrollo del modelo, bajo el entendido de que la investigación social implica un camino largo, lleno de dificultades, tan sustantivos como lo que se pretende resolver (Briones, 1991).

Este trabajo de investigación se justifica también, porque está orientado a incentivar que las investigaciones que se produzcan en la Unidad de Postgrado de la Facultad de
Economía de la UMSA aporten efectivamente a la estabilidad y desarrollo de la Economía Nacional, promoviendo un clima favorable a la investigación científica en la Facultad de Economía y todos sus estamentos.

\section{METODOLOGÍA}

Se trató de un estudio cualitativo de corte transversal, con alcance exploratorio y descriptivo, que en una primera instancia se enfocó en demostrar la factibilidad de un modelo matricial de investigaciones académicas, mediante la exploración y análisis de variables cualitativas que permitieron examinar la solución de diversas necesidades detectadas en la universidad boliviana. Posteriormente se propusieron las condiciones operativas necesarias para el Modelo.

El diseño experimental está estrechamente relacionado con el problema de investigación y el tratamiento del estudio de caso descrito y focalizado en los potenciales investigadores que han culminado sus programas de postgrado, maestrías y doctorados, en la Facultad de Economía de la UMSA.

Se eligió como unidad de análisis precisamente la Unidad de Postgrado de la Facultad de Economía de la UMSA, en la que se identificaron interesados y beneficiarios directos, que son los estudiantes de los distintos programas de postgrado en Economía, actuales y potenciales, específicamente en la etapa de la investigación científica (Tesis), y también agentes relacionados conformados por autoridades y profesores.

El diseño metodológico de la investigación estuvo definido y respaldado por 
el método analítico-sintético y el alcance fue principalmente exploratorio, aunque también fue descriptivo, habida cuenta que se exploró la propuesta de un Modelo que se acompaña con técnicas e instrumentos de recolección de información (consulta a expertos, encuesta estructurada, entrevista a profundidad).

Se aplicó la consulta a expertos, con el fin de explorar y analizar los criterios que el modelo matricial de investigaciones académicas macroeconómicas debería considerar para, mediante un enfoque interdisciplinar y de complementariedad, aportar al diseño de Políticas Públicas. El equipo de expertos estuvo conformado por un representante de la Cámara Nacional de Industria, otro de la Cámara Nacional de Comercio, el director de la Unidad de Postgrado de la Facultad de Economía de la UMSA y dos economistas destacados por sus aportes en la elaboración de Políticas Públicas de Bolivia. La encuesta estructurada se aplicó para recolectar información de los estudiantes egresados de Postgrado que tienen pendiente la elaboración de la tesis y están habilitados para iniciar este proceso. Finalmente, se realizó una entrevista que recogió la percepción de 20 docentes eméritos de esta Universidad, los principales criterios para la selección de los docentes fue su trayectoria académica, experiencia profesional en áreas de investigación y macroeconomía y su decisión de cooperar en el estudio.

Según se muestra en la tabla 1 , se plantearon cinco ejes temáticos relativos a la implementación del modelo de investigaciones académicas macroeconómicas, que fueron el marco de referencia para la aplicación de los tres instrumentos citados líneas arriba. Estos ejes temáticos, han permitido detectar las condiciones operativas necesarias para el Modelo.

Tabla 1. Ejes temáticos basados en el concepto de tesis simultáneas, complementarias y relacionadas.

\section{ET}

ET-1 Oferta de temas investigativos seleccionados por un Comité de Investigación de la Facultad de Economía UMSA.

ET-2 Impulsar la titulación de los postulantes rezagados mediante el trabajo de investigación (tesis).

ET-3 Orientar la investigación (tesis) al aporte a Políticas Públicas.

ET-4 Apalancar los recursos con el aporte de todos los involucrados en la investigación.

ET-5 Vincular Universidad y Estado a través de la investigación académica. 
Las condiciones operativas necesarias para el Modelo se definieron considerando los ejes temáticos presentados en la tabla 1 y los aportes y criterios de científicos contemporáneos que se citan a continuación: (1) Álvarez-Gayou (2003), que postula que la ciencia es compleja y heterogénea, por lo que no es posible aplicar un solo método en todas las disciplinas; (2) Strauss y Corbin (2002), que consideran que el investigador tiene una experiencia vivida múltiple, fruto de la literatura de la disciplina científica de su profesión; (3) Arellano (1979), afirma que el valor social sustantivo en el que se debe enfocar una investigación es ayudar a mejorar la calidad de vida de los demás, en lo económico, psicológico, ambiental, político o intelectual; (4) Zorrilla (1994), enfatiza en la necesidad de aplicar enfoques multidisciplinarios, interdisciplinarios y de complementariedad; (5) Gómez (2011), argumenta que toda universidad debe contar con un sistema organizacional de investigación; (6) Ander-Egg (1974) y Briones (1991), advierten que la investigación social implica un camino largo, lleno de dificultades, tan complejas como los problemas que pretenden resolver.

Por otra parte, la técnica de la encuesta consideró a la población de Postgrado que no culminó sus trabajos de investigación de tesis para obtener la acreditación, la población de habilitados para iniciar sus investigaciones fue de 4.262 postulantes, hasta el 30 de septiembre de 2020 .

Se recolectó la información con la aplicación de una encuesta estructurada, enmarcada en los 5 ejes temáticos previamente explicados, permitiendo averiguar e interpretar la aceptación, rechazo o respuesta dispersa de esta población. La medición de los datos se ha efectuado a través de promedios ponderados, para dimensionar la concentración de las percepciones y respuestas comunes. Para el cálculo de la muestra estadística representativa, se utilizó la expresión matemática:

$$
n=\frac{N * G^{2}(P * Q)}{E^{2}(N-1)+G^{2}(P * Q)}
$$

Dónde:

$n=\quad$ Tamaño de la muestra.

$N=$ Población Total

$G=$ Nivel de confianza (que es $95 \%$, equivalente a 1,96)

$E=$ Margen de error (que es de un $5 \%$, equivalente a 0,05 )

$P=\quad$ Valor esperado del universo (50\% del universo, equivalente a 0,5 )

$Q=$ Valor esperado del universo (50\% del universo, equivalente a 0,5 )

El universo en esta etapa estuvo conformado por 4.262 postulantes de Postgrado que no iniciaron sus trabajos de investigación de tesis. La muestra probabilística necesaria para obtener un nivel de confianza del 95\% debió estar compuesta por 400 postulantes. Sin embargo, dada la apertura de los estudiantes de Postgrado, a participar de la misma y para tener una muestra más representativa, se decidió consultar a 560 postulantes.

RESULTADOS

Para la mejor comprensión de los resultados obtenidos, este apartado se divide a 
su vez en cinco subtítulos: a) Criterios para el modelo matricial de investigaciones académicas macroeconómicas, b) Hallazgos obtenidos en la consulta a los postulantes, c) Estimación de recursos necesarios para el desarrollo del modelo, d) Operación y funcionamiento del Modelo y e) Condiciones operativas necesarias para el Modelo.

\section{Criterios para el modelo matricial de investigaciones académicas macroeconómicas}

Se presentan los resultados de la consulta a expertos desglosando el análisis suscitado en torno a los cinco ejes temáticos establecidos.

\section{ET-1. Oferta de temas investigativos} seleccionados por un Comité de Investigación

Respecto a este eje temático, el análisis derivó en lo siguiente: (1) La necesidad de establecer criterios de pertinencia, que deriven en lineamientos para la selección de temas de investigación; (2) Las líneas de investigación en macroeconomía deberán dar lugar el estudio sistémico, con criterios de alcance exploratorio, descriptivo, relacional y/o explicativo, que describen en una situación real, para concluir en un aporte concreto al desarrollo de Políticas Públicas; (3) La investigación científica, llegará desde lo general, producto de la primera etapa de la observación, a lo especifico, para deducir modelos, paradigmas, leyes, teoremas, metodologías, modelos, procesos y procedimiento enfocados a dar solución a los diversos problemas económicos de la sociedad, tanto de escasez, como de exceso; (4) La matriz deberá implementarse bajo un enfoque sistémico, que considere "el todo" (en este caso el problema macro relacionado con la economía), compuesto por partes, interdependiente, interrelacionadas e interactuantes entre sí (en este caso cada parte podrá enfocarse en una necesidad específica que deberá ser atendida por cada tema de investigación), en el análisis de entes y temáticas divergentes y complementarias; (5) La matriz asume como importante referencia los siete saberes necesarios para la educación de Morín (1999): Las cegueras del conocimiento, los principios de un conocimiento pertinente, enseñar la condición humana, enseñar la identidad terrenal, enfrentar las incertidumbres, enseñar la comprensión y, por último, la ética del género humano del pensamiento complejo. Morín induce a la epistemología científica, el pensamiento interdisciplinar, multidisciplinar y transdisciplinar, y el trascender del positivismo, hacia la fenomenología, con un espíritu de análisis integral de la sociedad, sus problemas y necesidades.

ET-2. Impulsar la titulación de los postulantes rezagados mediante la elaboración de la Tesis

Se pudo evidenciar que está generalizada la percepción de la necesidad del apoyo al proceso de creación de las tesis, ya que se considera que la estructura académica y administrativa de las organizaciones dedicadas a la educación superior, la disciplina, el ambiente ético y de búsqueda de conocimiento e innovación, son la base y han sido terreno fértil para aplicar la investigación y 
orientarla a encontrar el método ideal para incrementar la calidad y cantidad de investigaciones que están relacionadas, respecto a un problema macroeconómico identificado, que afecta e impacta negativamente en la sociedad y su desarrollo armónico acelerado. Por estas razones, los potenciales tesistas rezagados, son un problema urgente por resolver.

\section{ET-3. Orientar la investigación al aporte a} Políticas Públicas

Como resultado de la consulta, se entiende la preocupación e importancia de: (1) Explorar las políticas y estrategias para establecer una iterativa relación entre los gobiernos nacionales y subnacionales, con la Universidad Pública especialmente para que se logre el aporte académico en el diagnóstico de problemas de la sociedad, que conduzcan a esta relación de cooperación al diseño y formulación técnica de Políticas Públicas que justifique la planificación estratégica y la ejecución de programas y proyectos de desarrollo económico. (2) La conformación de equipos técnicos, para la definición y ejecución de estudios de línea de base referidos a variables de desarrollo económico y social.

Eje temático 4. Apalancar los recursos con el aporte de todos los involucrados en la investigación

$\mathrm{Si}$ se parte de la evidente escasez de recursos para investigaciones académicas, entonces se debe explorar otros recursos, bajo el concepto moderno de apalancamiento financiero, que no se refiere únicamente al crédito, sino a inyectar recursos a menor costo financiero a los proyectos de investigación.

En este eje temático, se consideraron el aportes posibles, al margen de su cuantía, de todos los involucrados, por ejemplo los provenientes de los estudiantes de pregrado encargados de participar en trabajos específicos para el desarrollo de las investigaciones, como las encuestas, su tabulación, el informe preliminar de los resultados que a cambio de su acreditación, en determinada asignatura, aportan con gastos de transporte, de material de escritorio y no son hechas con personal contratado, sino más bien invitado y la participación de docentes como guías de la organización, vaciado y tabulación de encuestas y elaboración de informes específicos de conclusiones.

Eje temático 5. Vincular Universidad y Estado a través de la investigación académica

En este eje temático se identificaron dos factores estratégicos: (1) La retroalimentación, para actualizar permanentemente los programas de formación académica, procurando que respondan a la realidad nacional. (2) La estructura académica y administrativa de las organizaciones dedicadas a la educación superior, el ambiente ético y de búsqueda de conocimiento e innovación, agregados al logro de y resultados concretos, son la base y han sido terreno fértil para aplicar la investigación y orientarla a encontrar el método ideal para incrementar la calidad y cantidad de investigaciones que están relacionadas, respecto a un problema macroeconómico identificado, que afecta e 
impacta negativamente en la sociedad y su desarrollo armónico acelerado.

\section{Hallazgos obtenidos en la consulta a los postulantes}

En la tabla 2 se aprecia una síntesis de las respuestas emitidas por la población objeto de estudio, en la que se puede observar los 5 ejes temáticos elegidos en la investigación base de este artículo y sus respuestas, considerando 3 alternativas; es decir, la respuesta positiva, la respuesta negativa, las respuestas dispersas o no concretas y sus porcentajes ponderados; lo que lleva a determinar la concentración de preferencias y percepciones de esta población.

Tabla 2. Sistematización de resultados obtenidos en la encuesta a postulantes

\begin{tabular}{ccccc}
\hline $\begin{array}{c}\text { EJE } \\
\text { TEMÁTICO }\end{array}$ & $\begin{array}{c}\text { Respuesta } \\
\text { positiva } \\
\text { RESP (+) }\end{array}$ & $\begin{array}{c}\text { Respuesta } \\
\text { negativa } \\
\text { RESP (-) }\end{array}$ & $\begin{array}{c}\text { Respuesta } \\
\text { difusa o } \\
\text { dispersa } \\
\text { RESP (?) }\end{array}$ & Matiz específico de la respuesta difusa \\
MATIZ
\end{tabular}

Los resultados presentados en la tabla 2 ratifican la importancia de plantear un modelo alternativo de investigaciones simultáneas, relacionadas y complementarias; sobre todo, para impulsar investigaciones pertinentes que aporten al diseño de Políticas Públicas e incrementen la producción de investigaciones en la Facultad de Economía de la UMSA.

En los cinco ejes temáticos propuestos se percibe claramente que los estudiantes de
Postgrado, potenciales investigadores para lograr su acreditación, privilegian la orientación de la investigación (tesis) al aporte a Políticas Públicas y están predispuestos a impulsar su titulación y evitar estar rezagados en titulación. Con relación al ET-1, al $86 \%$ le parece importante recibir una oferta de temas investigativos seleccionada por un Comité de Investigación de la Facultad de Economía UMSA, el restante $14 \%$ prefiere que no sea la 
única fuente de temas de investigación. Respecto al ET-2, el $71 \%$ de la población potencial de investigadores, está de acuerdo con asumir la modalidad de tesis en la etapa de titulación, el restante $23 \%$ está en desacuerdo y el $6 \%$ sugiere que no sea la única opción.

En ET-4, de apalancamiento de recursos con el aporte de todos los involucrados en la investigación, existe pesimismo en más del $21 \%$ de la población, a no ser que se encuentre una estrategia operativa, respaldada por políticas impulsadas y definidas específicamente por las autoridades de la Facultad de Economía, al respecto.

Donde se ha notado 94\% de aceptación y, sólo $6 \%$ de la población demuestra escepticismo, es en el eje temático referido a la vinculación de la Universidad y Estado a través de la investigación académica (ET-5). Son pocos los que no creen factible que las autoridades políticas nacionales $y$ subnacionales, tengan un interés real de ajustar sus propuestas y su respectiva ejecución, a la necesidad técnicamente diagnosticada.

Para contrastar el criterio de los estudiantes respecto al Modelo, se hizo una entrevista a docentes dispuestos a cooperar en el proceso de implantación del modelo. Los resultados obtenidos se resumen así: 1) En cuanto al ET-1, el $100 \%$ de los docentes creen que la universidad debe orientar y asesorar a los potenciales investigadores en la selección de sus temas, especialmente respecto al aporte a la sociedad y en el caso presente, al diagnóstico de problemas y sus propuestas de soluciones reales, a través de las tesis; 2) Respecto al ET-2, 80\% aprueba el modelo y cree que motivará la producción de investigaciones, e impulsará a la creación de una política de disminución de estudiantes rezagados, por otra parte, el $20 \%$ sugiere que no sea la única modalidad de investigación, es decir que existan varias modalidades alternativas, incluida la Matriz propuesta; 3) En cuanto al ET-3, 95\% de docentes cree posible y necesaria la orientación de tesis a Políticas Públicas, 5\% opinan que dependerá de la gestión de la dirección académica; 4) El análisis en torno al ET-4 muestra que $85 \%$ de los docentes cree que la propuesta es para el mediano y largo plazo, dependiendo del apoyo de las autoridades al modelo, incluyéndolo en las normas, con sus respectivos reglamentos y protocolos para todos los participantes de los distintos estamentos universitarios, el restante $15 \%$ califica al Modelo como atemporal, es decir, podría ser incorporado en cualquier tiempo e inclusive circunstancia; 5) Finalmente, con relación al ET-5, 60\% cree en la posibilidad de vinculación Universidad-Gobiernos, a través de estrategias consensuadas, como el dialogo y el trabajo sistémico en equipo, $40 \%$ condiciona la relación al establecimiento de una Políticas de Estado, que establezca una relación permanente y obligatoria de los gobiernos nacionales y sub nacionales con las universidades públicas, desde el diagnostico de los problemas y necesidades sociales, por ejemplo, en el establecimiento de líneas de base, respecto a variables macroeconómicas sustantivas, respecto a pobreza, salud, educación, servicios básicos y otros.

\section{Operación y funcionamiento del Modelo}

En el gráfico 1 se puede observar la matriz estructurada en un sistema de coordenadas, en 
el eje de abscisas se ubican los expertos que en sentido horizontal van asesorando en su especialidad cada una de las investigaciones comprendidas en la matriz, las cuales ingresas en el eje de las ordenadas como investigaciones numeradas (Investigación 1, Investigación 2, etc.).

\section{Gráfico 1.}

Estructura Matricial basada en el concepto de estructura matricial de gestión de proyectos

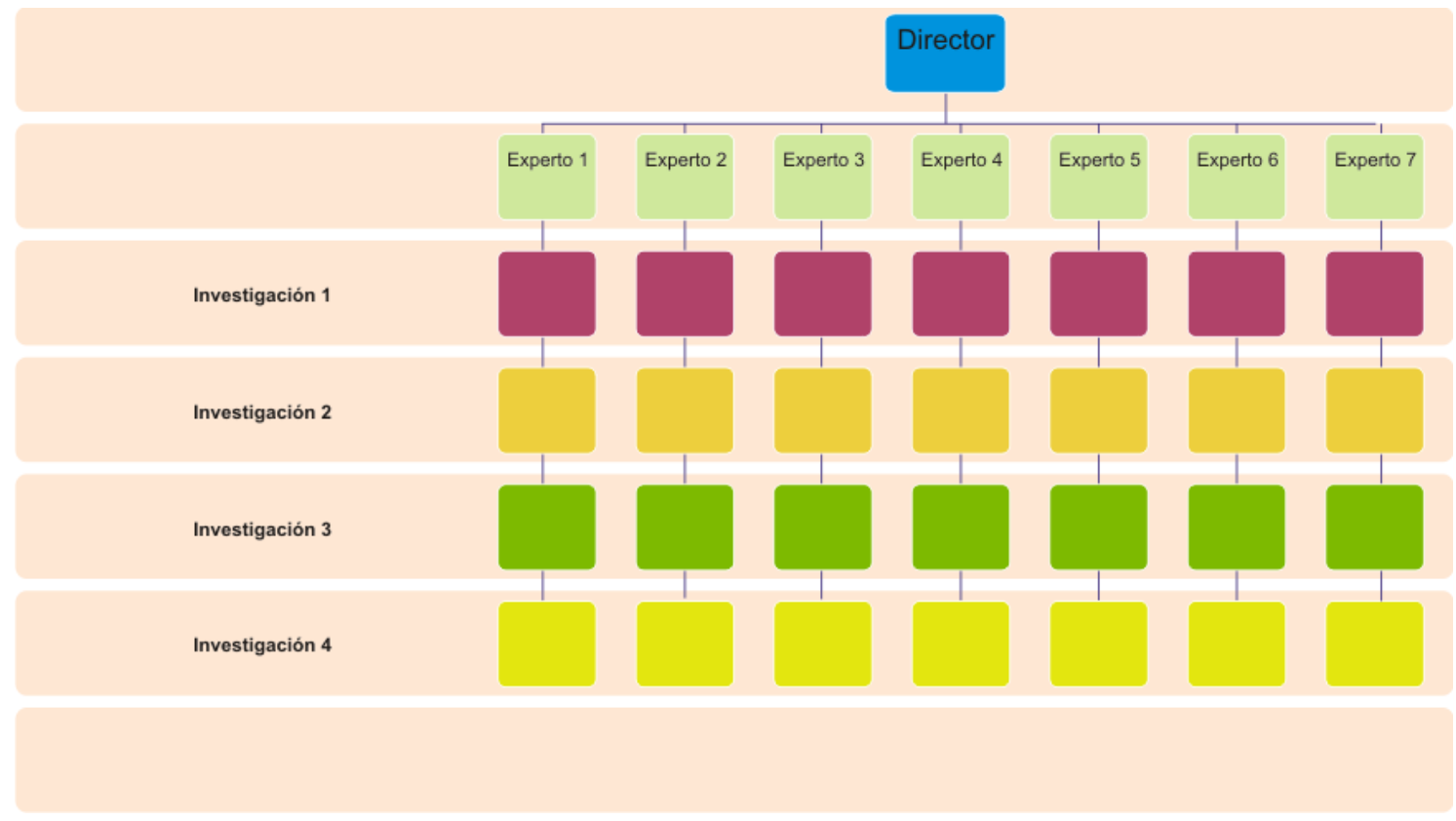

En el gráfico 1, en el título Investigación 5 (Fila 7), las columnas vacías sugieren la posibilidad de incluir más investigaciones relacionadas a la matriz.

La estructura matricial propuesta opera según se explica:

- El trabajo en equipo de los expertos que asesoran cada una de las investigaciones es simultáneo, debido a que están relacionadas respecto a un tema sustantivo de carencia o exceso detectado, tienen un enfoque multidisciplinario y sistémico, es decir, que confluyen interdisciplinariamente, con enfoque de complementariedad, en la búsqueda de un análisis y estudio integral y sistémico de las diferentes problemáticas macroeconómicas presentadas, y que se dirigen a aportar con soluciones también integrales y sistémicas, logrando objetivos comunes.

- Los expertos que actúan en el equipo lo hacen en condición de tutores que conforman un grupo multidisciplinario en las investigaciones, y son necesariamente seleccionados por su capacidad, 
conocimientos y experticia. El sentido de pertenencia funcional de grupo permite desarrollar un ambiente de trabajo científico colaborativo.

- Uno de los requisitos de la conformación de grupos de expertos, es su compromiso individual, es decir, la responsabilidad profesional que sumada da lugar a la responsabilidad de grupo, para lograr resultados tangibles del modelo.

- Se ejecutan varias investigaciones relacionadas. bajo la gestión y administración de cada uno de los investigadores que trabajan en sus tesis, pero necesariamente, cuentan con asesoramiento iterativo de un grupo multidisciplinario de expertos tutores con diversas especialidades, en relación directa con las disciplinas concurrentes en las investigaciones simultáneas.

- El principal supuesto facilitador para el funcionamiento de una estructura matricial, para la gestión de proyectos de investigación se concentra en los equipos que se conforman y se caracterizan por su flexibilidad. Se orientan a los objetivos y metas de los proyectos de investigación, permitiendo la selección adecuada de expertos en los objetos de estudio determinados. Del mismo modo, se identifica un supuesto letal que es acuerdo de ámbitos de acción y responsabilidades, se logra, siempre que entre los participantes e involucrados haya sido desarrollado un proceso de negociación ganar/ganar, que se debe tomar en cuenta también en la formación de los equipos de trabajo.

\section{Condiciones necesarias para la implementación del Modelo}

Las condiciones necesarias identificadas son:

La conformación de un Comité Académico de la Alta Dirección de la Organización Académica de Educación Superior (Universidad y su Consejo Superior) que es el encargado de seleccionar una investigación, destinada a solucionar un problema relacionado a un tema macroeconómico detectado en el país.

El problema sectorial, es dividido, fraccionado lógica y complementariamente $y$ con enfoque sistémico, tomado en cuenta además los paradigmas multidisciplinar e interdisciplinar del pensamiento complejo, desde su identificación, en investigaciones sub sectoriales y relacionadas, con objetivos mucho más concretos y específicos, pero estrechamente vinculados con los objetivos técnicos y de gestión de la investigación sectorial principal.

El proceso de licitación o puja es abierto. Los temas sub sectoriales producto del fraccionamiento en investigaciones complementarias resultantes relacionadas, son ofrecidos a los estudiantes de Postgrado, potenciales investigadores, que han cumplido con todos los requisitos académicos exigidos para llegar a esta instancia. Si alguna de las investigaciones sub sectoriales relacionadas, requiere experticia particular y específica, se incorpora al experto idóneo necesario.

Según se observó en la matriz expuesta en el gráfico 1, los expertos se ubican gráficamente en el eje de abscisas, situados con lógica 
sistémica secuencial, respecto al Modelo de Investigación propuesto; es decir, su trabajo es transversal de arriba hacia abajo, aportando con su experticia, todas y cada una de las investigaciones afines en curso. Lógicamente, el primer experto que empezará a recibir y a trabajar con las investigaciones será el profesional en metodología de la investigación científica. El segundo podría ser el experto en el tema de investigación, el tercero en sistemas informáticos, el próximo es experto en estadística aplicada, y los siguientes son expertos en economía y desarrollo, econometría, planificación estratégica y otras disciplinas vinculadas con el tema y sus alcances. Las investigaciones afines se ubican en el eje de las ordenadas, ingresan simultáneamente y recorren horizontalmente, pasando por cada experto, hasta llegar al último, arrojando un producto final.

Las investigaciones tienen sus responsables (propietarios), producto de la licitación de temas sub sectoriales, es decir son los propios postulantes a los que se les ha asignado cada una de las investigaciones, para su acreditación individual. Las investigaciones ingresan a la matriz, y van pasando a consideración de los expertos horizontalmente, hasta su finalización.

Estas investigaciones sub sectoriales concluidas, que responden y se originan en una macro investigación sectorial, son pasadas a un Comité Científico de la Universidad, para su revisión e integración final, orientada al problema macroeconómico, seleccionado en el inicio y fraccionado en temas específicos. El producto final, en primera instancia se entrega a las autoridades universitarias que, a su vez, las harán llegar a los poderes Ejecutivo y al
Legislativo en actos públicos y con la prensa especializada, para motivar el control social.

En este proceso operativo y funcional de la investigación, se ha congregado la participación y movilización no solo de la Unidad de Postgrados, sino también de todos los niveles académicos de la Facultad de Economía de la UMSA, empezando por los estudiantes de pregrado, los cuales a cambio de crédito para aprobar asignaturas $\mathrm{y} / \mathrm{o}$ cumplir requisitos exigidos, aportan bajo una guía, a las investigaciones, desde tareas operativas sencillas. Por ejemplo: a los estudiantes de mercadeo y comercialización, se les encarga el levantamiento de encuestas y con la guía de su profesor, proceden a la tabulación y emisión de conclusiones preliminares a ser incluidas en un informe, como insumo para el responsable de la investigación.

\section{CONCLUSIONES}

A través del desarrollo de la investigación y especialmente en el trabajo de campo, se ha evidenciado la factibilidad de ejecución, operatividad y flexibilidad de adaptación al entorno, mostrando un Modelo que permite incrementar la cantidad, pertinencia y calidad de investigaciones científicas necesarias para orientar las Políticas Públicas del sector de la economía nacional, maximizando el beneficio y la gestión de los recursos actuales y potenciales, destinados a la investigación científica académica y que funciona cumplimiento los siguientes objetivos específicos:

- Incentiva en la creación, concientización y consolidación de un ambiente favorable a la investigación científica, en todos los ámbitos 
y estamentos de la Faculta de Economía de la UMSA, con enfoque interdisciplinario y de complementariedad, promovido y liderado por el nivel de Postgrado. Implica el involucramiento, el aporte y el compromiso de todo el equipo, en relación con su nivel de preparación y formación alcanzados.

- Se orienta a diagnosticar la necesidad del establecimiento de una continua y permanente relación bilateral de cooperación con los gobiernos nacionales y subnacionales en tanto Política de Estado, para la producción de investigaciones académicas dirigidas al Sector de la Economía Nacional.

Se han identificado las siguientes necesidades operativas: (1) El compromiso de la Alta Dirección de la Institución de Educación Superior, en la que se implementa; (2) La selección del asunto temático de la investigación principal, (Investigación guía y/o Investigación Macro), debe estar a cargo de una Comité Especial de Investigaciones, que establezca interfaces de comunicación con las autoridades de gestión pública a nivel de toma de decisiones, para validar e identificar la pertinencia de los temas a investigar; (3) La disciplina en el cumplimiento de un Plan Estratégico para ejecutar las investigaciones es la ruta para evitar dificultades y motivar un clima favorable al trabajo en equipo y su focalización en los objetivos de la investigación principal y las investigaciones subyacentes específicas; (4) Es necesaria la socialización, explicación, concientización y capacitación de la propuesta del Modelo, en todos los estamentos de la Universidad, para informar correctamente y motivar un ambiente favorable a la investigación científica institucional; (5) Debe existir compromiso de trabajo en equipo, donde la coordinación, y responsabilidad, aseguren la calidad de los productos intermedios, constituidos en materia prima de las etapas superiores y más avanzadas de la investigación; (6) La Institución de Educación Superior, debe garantizar y cumplir con la ejecución presupuestaria oportuna de los recursos, especialmente los destinados a la remuneración del equipo de expertos nominados y otros; (7) El ambiente institucional favorable a la investigación científica y la armonía operatividad de los equipos, es un requisito indispensable, aspecto en el que se debe trabajar prioritariamente.

La pertinencia de otras líneas de investigación en torno a la propuesta de factibilidad de un modelo matricial de desarrollo de investigaciones relacionadas y simultáneas es perfectamente posible. Asimismo, es factible su aplicación en otras Facultades o instituciones de Educación Superior. Finalmente, este estudio deja sobre la mesa la necesidad de la formulación de Políticas de Estado con relación a establecer un puente de comunicación y cooperación continua entre el Estado, representado por los gobiernos nacionales y subnacionales y las Universidades.

\section{REFERENCIAS}

Álvarez-Gayou, J. L. (2003). Cómo hacer investigación cualitativa: Fundamentos y metodología. México: Paidós Educador

Ander-Egg, E. (1974). Introducción a las técnicas de investigación social. Buenos Aires: HumanitasAnguera, M. T. (1996). Metodología de la observación de las ciencias humanas. San José: EUNED 
Arellano G, J. (1979) Elementos de investigación: La investigación a través de su informe. San José: EUCR

Bosque-Rodríguez (1998). Investigación Elemental. México: Trillas

Briones, G. (1991). La investigación social y educativa. Caracas: Convenio Andrés Bello

Gómez S, G. (2011). Cinco Tesis sobre investigación científica en la Universidad Politécnica de Nicaragua. Recuperado de http://biblioteca.clacso.edu.ar/Nicaragua/ cielac-upoli/20170823053728/Cinco-tesissobre-investigacion.pdf

Lases, M. A. \& Robles M. A. (2008). Manual Elemental de Proyectos de Investigación. México: Centro de Impresión Digital Lases
Morín, E. (1999). Los siete saberes necesarios para la educación del futuro. Editorial Santillana. Medellín

Strauss, A., \& Corbin, J. (2002). Bases de la investigación cuantitativa. Técnicas y procedimientos para desarrollar la teoría fundamentada. Medellín. Colombia: Editorial Universidad de Antioquia

Vargas C, Z. R. (2009). La investigación Aplicada, una forma de conocer realidades con evidencia científica. Educación, 33(1), 155165

Zorrilla A, S. (1994). Introducción a la Metodología de la Investigación. México: Editorial Aguilar León 